\title{
Artifact peaks due to minor decomposition of sample diluent DMSO in head space-GC analysis: Mass spectrometric investigation using deuterated DMSO and Quadrupole Time-of-Flight mass detection
}

\author{
Jinsheng Lin ${ }^{\mathrm{a}}$, Qiang Zhou ${ }^{\mathrm{a}}$, Jun Wang ${ }^{\mathrm{b}}$, Dandan Tang ${ }^{\mathrm{c}}$, Wenquan Zhu ${ }^{\mathrm{a}, \mathrm{d}}$, \\ Wenbin Chen ${ }^{\mathrm{a}}$, Qiangming $\mathrm{Li}^{\mathrm{c}}$, Yan $\mathrm{Zhu}^{\mathrm{b}}$, and Min $\mathrm{Li}^{\mathrm{a}, \mathrm{e},}$, \\ ${ }^{a}$ Center of Excellence for Modern Analytical Technologies (CEMAT) \\ Huahai Pharmaceutical Co., Ltd, Xunqiao, Linhai, Zhejiang, 317024, P.R. China. \\ ${ }^{b}$ Quality Control Department of Chuannan Site, Zhejiang Huahai Pharmaceutical Co. \\ Ltd., Duqiao, Linhai, Zhejiang, 317016, PR China. \\ ${ }^{c}$ Quality Control Department of Xunqiao Site, Zhejiang Huahai Pharmaceutical Co. \\ Ltd., Xunqiao, Linhai, Zhejiang, 317024, PR China. \\ ${ }^{d}$ Quality Research Department of API, Zhejiang Huahai Pharmaceutical Co. Ltd., \\ Xunqiao, Linhai, Zhejiang, 317024, PR China. \\ ${ }^{e}$ Huahai US, Inc. 700 Atrium Drive, Somerset, NJ 08873, USA.
}

\begin{abstract}
Rationale: Head space gas chromatography (HS-GC) has become a widely used analytical technique for residual solvents determination in drug substances and drug products. During our method development of residual solvents by HS-GC for certain drug substances, four unknown peaks were observed when dimethyl sulfoxide (DMSO) was employed as sample diluent.
\end{abstract}

Methods: Use of other sample diluents replacing DMSO did not show these unknown peaks in the HS-GC analysis. HS-GC-MS was then utilized to investigate these peaks. As part of the investigation, DMSO- $d_{6}$ was used to trace the origins of these unknown peaks to DMSO. A stress study of methanethiol and formic acid was carried out to provide further evidence that $S$-methyl methanethioate was one of the unknown peaks observed during the original HS-GC analysis.

Results: The four unknown peaks have been identified as methanethiol, 
dimethylsulfide, $S$-methyl methanethioate, and dimethyldisulfide, respectively, and it has been demonstrated that the four peaks are clearly related to DMSO through the use of its deuterated counterpart. Plausible formation mechanisms of these species are proposed.

Conclusions: The occurrence of the peaks is due to minor degradation of DMSO, facilitated by certain drug substances as analytes of the analysis. These peaks are artifacts, rather than genuine impurities of the drug substances. These artifact peaks could widely occur in HS-GC analysis, when DMSO is used as sample diluent.

Key words: Headspace GC; GC-MS; false positive; DMSO decomposition; stable isotope labeling

\section{Introduction}

According to the International Conference on Harmonisation of Technical Requirements for Registration of Pharmaceuticals for Human Use (ICH) guidelines of Q3C, impurities that are related to active pharmaceutical ingredient (API) can be classified into three categories, i.e., organic impurities, inorganic impurities, and residual solvents. ${ }^{1}$ Residual solvents may be introduced from the starting materials or solvents used in the manufacturing process. ${ }^{2-6}$ As there is no therapeutic benefit from residual solvents, all residual solvents should be treated as process impurities and must be removed to the extent to meet the product specifications, good manufacturing practices (GMP), or other quality-based requirements. ${ }^{1}$

The commonly used analytical technique for residual solvents determination in 
drug substances and drug products is gas chromatography with headspace sampling (HS-GC analysis). ${ }^{7-10}$ Due to its good solubility for vast majority of organic compounds, DMSO is widely used as diluent for sample preparation in the GC and LC analysis, ${ }^{11-14}$ and it is also used as sample diluent in residual solvents analysis for a large number of Huahai's drug substances.

In our residual solvents analysis of several drug substances with HS-GC methods using DMSO as the diluent, four minor unknown peaks were observed. It is worth to mention that two of these unknown peaks could also be found in the blank injections, albeit their amounts were much less than those occurred in the sample injections, as evidenced by their timid signal intensities that were less than $\sim 1 / 10$ of those observed in the sample injections. Meanwhile, the other two peaks were mostly not detected in the blank solution (the detection limit: $0.1 \mathrm{ppm}$ ). On the other hand, they could not be found in HS-GC analysis of the same drug substance sample when DMSO is replaced by DMF as the diluent.

In this paper, we demonstrate that the occurrence of the above four unknown peaks is due to the thermal instability of DMSO in the presence of certain drug substances under the relatively high temperature during the headspace sampling process (usually 80 to $140^{\circ} \mathrm{C}$ ). Hence, these four peaks are artifact peaks, or ghost peaks, rather than genuine impurities of the drug substances. Further investigation shows that these artifact peaks can occur with other structurally diverse drug substances, indicating this could be a widely occurred phenomenon in HS-GC analysis, when DMSO is used in the sample diluent. Based on our literature search, it 
seems there has been no systematic study to clearly implicate artifact peaks in HS-GC analysis, other than a recent paper published by our group (in which formaldehyde was found to be an artifact peak). ${ }^{15}$ With the ever tightening regulatory requirements, small unknown peaks observed in GC analysis may need to be scrutinized for the purpose of risk assessment. The results of this paper should facilitate the root cause investigation, should such events occur in other pharmaceutical laboratories.

\section{Experimental}

\subsection{Materials}

DMSO and DMF ( $N, N$-dimethylformamide) of HPLC grade were purchased from ROE Scientific Inc. (Newark, USA). DMSO- $d_{6}$ was procured from TCI (Shanghai, China). Formic acid of HPLC grade was purchased from Merck KGaA (Darmstadt, Germany). Methanethiol (10\% v/v in toluene) was a product by Sigma-Aldrich Corp. (St. Louis, MO, USA). Acetic anhydride was supplied by Hangzhou Chemical Reagent Co., Ltd (Hangzhou, China). GC headspace bottles and bottle caps were products of Agilent Corp. (Santa Clara, CA, USA). All other reagents were from various vendors. All the drug substances used in this study are products of Huahai Pharmaceutical, Co. Ltd.

\subsection{Unit resolution $H S-G C-M S$ analyses}

The HS-GC-MS analyses were performed on an Agilent 7890A gas chromatograph coupled with a 5975C quadrupole mass spectrometric detector and a 7697A headspace sampler (Agilent Technologies, Palo Alto, CA, USA). The loop and transfer line were operated at $110^{\circ} \mathrm{C}$ and $115^{\circ} \mathrm{C}$, respectively. The oven temperature was set at $80^{\circ} \mathrm{C}$ and 
the thermal equilibration time was $45 \mathrm{~min}$. The headspace sampler was vibrated at a speed of 18 shakes per min during sampling. The injection temperature was set at $140^{\circ} \mathrm{C}$. The injection time was $1.0 \mathrm{~min}$ and the injection volume was $1.0 \mathrm{~mL}$. The chromatographic separations were performed on an Agilent DB-624 capillary GC column (30 $\mathrm{m} \times 0.32 \mathrm{~mm} \times 1.8 \mu \mathrm{m}, \mathrm{J} \& \mathrm{~W}$ Scientific). The GC oven temperature was programmed from an initial temperature of $40^{\circ} \mathrm{C}$ held for $15 \mathrm{~min}$, ramped at $20^{\circ} \mathrm{C} / \mathrm{min}$ to $140^{\circ} \mathrm{C}$ and then held for $15 \mathrm{~min}$. High-purity helium was used as the carrier gas at a constant flow rate of $1.0 \mathrm{~mL} \mathrm{~min}{ }^{-1} .1 \mathrm{~mL}$ of headspace gas was injected in split mode (split ratio $2: 1$ ) at $140^{\circ} \mathrm{C}$. Helium was used as carrier gas at $5.0 \mathrm{~mL} / \mathrm{min}$, split injection mode with a split ratio of 4:1 was employed. The total run time was 35.0 min with a solvent delay of 3.0 min. The mass spectrometer was operated with electron ionization $(70 \mathrm{eV})$ in full mass scan mode. The ion source temperature and quadrupole temperature were set at $230^{\circ} \mathrm{C}$ and $150^{\circ} \mathrm{C}$, respectively. Mass scan range was set from $\mathrm{m} / \mathrm{z} 33$ to 500 . The relative voltage and EMV were set at $200 \mathrm{~V}$ and 1,400 V, respectively. Data acquisition and processing was performed using ChemStation software.

\subsection{High resolution HS-GC-QTOF MS analyses}

The high resolution HS-GC-MS analyses were performed on an Agilent 7890A gas chromatograph coupled with a G7250 QTOF (quadrupole time-of-flight) mass spectrometric detector and a 7697A headspace sampler (Agilent Technologies, Palo Alto, CA, USA). The GC chromatographic conditions were the same as those of the unit resolution HS-GC/MS (single quadrupole MS) analyses listed in Section 2.2. The 
mass spectrometer was operated with electron ionization $(70 \mathrm{eV})$ in full mass scan mode. The ion source temperature and quadrupole temperature were set at $200^{\circ} \mathrm{C}$ and $150^{\circ} \mathrm{C}$, respectively. Mass scan range was set from $\mathrm{m} / \mathrm{z} 33$ to 500 . The resolution ratio was 12,500 and peak width was set at $0.7 \mathrm{sec}$. The emission current was $2.65 \mu \mathrm{A}$ and the acquisition rate was set at 5 spectra/s. Data acquisition and processing was performed using Mass Hunter software.

\subsection{Sample preparation}

Certain volume of diluent (regular DMSO) was transferred to GC headspace bottles. Afterward, the drug substances were added to these bottles respectively and shaken to dissolve to give the final concentration of $\sim 200 \mathrm{mg} / \mathrm{mL}$; the prepared samples were analyzed with the methods listed in Section 2.2.

2.5 Replacement of regular DMSO by DMF or DMSO-d for sample preparation in $H S-G C$ and $H S-G C-M S$ analyses

In order to confirm that the four unknown peaks originate from the diluent DMSO, other solvents such as DMF or DMSO- $d_{6}$ were used to replace the regular DMSO in the sample preparation of drug substances. The prepared samples were analyzed with the methods listed in Sections 2.2 and 2.3.

\subsection{Stress study of methanethiol and formic acid in DMF}

A stress study was designed to confirm if the unknown peak eluting at $\sim 17.0$ min (peak-3) is $S$-methyl methanethioate. Considering the fact that $S$-methyl methanethioate may be generated via condensation between methanethiol and formic acid, a forced degradation or stress study of formic acid and methanethiol in DMF 
was designed to help confirm the identity of the peak: to $3.0 \mathrm{~mL}$ of DMF was added $1.0 \mathrm{~mL}$ of formic acid and $1.0 \mathrm{~mL}$ of methanethiol solution $(10 \% \mathrm{v} / \mathrm{v}$ in toluene), respectively. After mixing, the resulting solution was loaded onto the two HS-GC-MS instrument for analysis with the methods listed in Sections 2.2 and 2.3.

\section{Results and discussion}

\subsection{HS-GC-MS analyses}

During our HS-GC-MS analysis of residual solvents in several drug substances, four minor unknown peaks were found when dimethyl sulfoxide (DMSO) was used as the sample diluent in the analyses. The four unknown peaks eluted at 5.704, 8.936, 17.011, and 20.800 min, which are labeled as peak-1, peak-2, peak-3 and peak-4, respectively (Fig. 1a). According to their mass spectra and the matching results against the NIST MS library, peak-1, peak-2 and peak-4 were readily identified as methanethiol, dimethylsulfide, and dimethyldisulfide, based on their high matching scores of 91\%, 95\%, and 97\% (Fig. 2a, Fig. 3a \& Fig. 4a), respectively. Nevertheless, peak-3 could not be readily identified, due to no matched hit for its mass spectrum in the NIST library. However, as shown in the mass spectrum of peak-3 (Fig. 5a), the molecular ion, which was also the base peak in the mass spectrum, exhibited $\mathrm{m} / \mathrm{z}$ at 76.0. A main fragment ion of peak-3 showed its $m / z$ at 47.0 , which could result from a loss of a formyl free radical (-29 Da) from the molecular ion. Meanwhile, two other fragment ions were observed with their $\mathrm{m} / \mathrm{z}$ at 48.0 and $\mathrm{m} / \mathrm{z}, 46.0$, respectively. It appears that these two fragments may result from the respective neutral losses of $\mathrm{CO}$ 
and HCHO (formaldehyde) from the molecular ion. Based on the structure of DMSO and its plausible degradation pathways (Scheme 1a), peak-3 was suspected to be $S$-methyl methanethioate.

All the identified or proposed structures of these four unknown species contain one or more sulfur atoms in their structures, suggesting that they may all derive from the same origin that contains sulfur. By examining the manufacturing processes of these drug substances, no sulfur-containing intermediates and reagents are used. Thus, it would not be possible that these four species originate from the drug substances, their intermediates, solvents or the starting materials. On the other hand, a few studies reveal that DMSO can decompose under extreme heating, microwaving, or advanced oxidation processing with hydroxyl radicals to produce a number of degradants including methanethiol and dimethylsulfide. ${ }^{16-21}$ Although these reported conditions are very harsh (e.g., pyrolysis at $\sim 800$ to $\sim 1400^{\circ} \mathrm{C}$ ) and thus are not relevant with regard to the pharmaceutical analytical condition utilized in the current study, it provided a reasonable basis to hypothesize that these four species could be generated from minor decomposition of DMSO during the HS-GC-MS analyses, particularly during the incubation period of the headspace sampler at $80^{\circ} \mathrm{C}$. In order to confirm this hypothesis, we replaced DMSO by DMF in the sample preparation of two of the drug substances with the same batches, while keeping all other parameters unchanged. It was not surprising to find that the four peaks were not detected (Fig.1c).

3.2 Replacement of DMSO by DMSO-d 6 in the sample preparation to help identify the origin of the four peaks 
In order to further verify that the above four peaks are derived from DMSO, regular DMSO was replaced by deuterated dimethyl sulfoxide (DMSO- $d_{6}$ ) as the sample diluent to dissolve the drug substances in HS-GC-MS analysis. As a typical example, the TIC chromatogram of one drug substance (using DMSO- $d_{6}$ as diluent) analyzed by HS-GC-MS displayed four target peaks at 5.663, 8.794, 16.929 and 20.709 min, respectively (Fig.1b); their retention times were consistent with the original elution profile of the four peaks $(5.704,8.936,17.011$, and $20.800 \mathrm{~min}$, respectively) in the HS-GC-MS analysis using regular DMSO as the diluent (Fig. 1a). Furthermore, the mass spectra of the four species clearly showed the expected deuterium mass shifts (refer to Fig. 2 through Fig. 5).

Specifically, for peak-1 (methanethiol, $\mathrm{CH}_{3} \mathrm{SH}$ ), its molecular ion of $m / z, 48$ was the second abundant peak in the MS spectrum (Fig. 2a); the corresponding deuterated molecular ion should have its $\mathrm{m} / \mathrm{z}$ at 52 , which was present but at a surprisingly low level (Fig. 2b). On the other hand, the $m / z, 51$ ion, which can be attributed to $\mathrm{CD}_{3} \mathrm{SH}^{+}$, became the most abundant ion in the GC-MS analysis when DMSO- $d_{6}$ was used as the diluent (Fig. 2b). This phenomenon may be explained by a plausible mechanism for the decomposition of DMSO in the HS-GC-MS analysis (Scheme 1): the decomposition would likely be trigged via an initial enolization-like process followed by 1,2-hydroxyl migration, to yield the presumed key intermediate of hydroxydimethylsulfoxide $\left(\mathrm{HO}-\mathrm{CH}_{2} \mathrm{SCH}_{3}\right)$. The latter intermediate has an exchangeable proton (the hydroxyl group); thus, in the case of using DMSO- $d_{6}$ as the diluent, the corresponding active deuterium of $\mathrm{DO}-\mathrm{CD}_{2} \mathrm{SCD}_{3}$ can exchange with the 
protons of residual water to produce $\mathrm{HO}-\mathrm{CD}_{2} \mathrm{SCD}_{3}$, which can decompose to $\mathrm{CD}_{3} \mathrm{SH}$, i.e., the species responsible for the $m / z 51$ ion. Furthermore, for the main fragment at $\mathrm{m} / z 47.0\left(\mathrm{CH}_{3} \mathrm{~S}^{+}\right)$and another major fragment at $\mathrm{m} / z 45.0\left(\mathrm{HCS}^{+}\right)$, their corresponding deuterated fragments were observed at $m / z 50.0\left(\mathrm{CD}_{3} \mathrm{~S}^{+}\right)$and $46\left(\mathrm{DCS}^{+}\right)$, respectively (Fig. 2a versus Fig. 2b). On the other hand, the fragment at $\mathrm{m} / \mathrm{z}, 33$, which should be attributed to ${ }^{+} \mathrm{SH}$, had no mass shift between the two experiments (Fig. $2 \mathrm{a}$ and $2 \mathrm{~b}$ ), as expected. Therefore, the observed mass shifts are consistent with the structure assignment of peak-1 as methanethiol.

For peak-2 (dimethylsulfide, $\mathrm{CH}_{3} \mathrm{SCH}_{3}$ ), its molecular ion of $\mathrm{m} / \mathrm{z} 62.0$ was the base peak in the mass spectrum (Fig. 3a) and its deuterated counterpart $\left(\mathrm{CD}_{3} \mathrm{SCD}_{3}\right)$ was observed at $\mathrm{m} / \mathrm{z}, 68.0$ as expected (Fig. 3b). For the two major fragments at $\mathrm{m} / \mathrm{z}$ $47.0\left(\mathrm{CH}_{3} \mathrm{~S}^{+}\right)$and $45\left(\mathrm{HCS}^{+}\right)$, their corresponding deuterated fragments were observed at $m / z 50.0\left(\mathrm{CD}_{3} \mathrm{~S}^{+}\right)$and $46\left(\mathrm{DCS}^{+}\right)$, respectively. Furthermore, the minor fragments at $\mathrm{m} / \mathrm{z} 35$ and 27 also displayed their corresponding deuterated counterparts. Hence, the observed mass shifts are consistent with the structure assignment of peak-2 as dimethylsulfide.

For peak-4 (dimethyldisulfide, $\mathrm{CH}_{3} \mathrm{SSCH}_{3}$ ), its molecular ion of $m / z, 94$ was also the base peak (Fig. 4a) and its deuterated counterpart $\left(\mathrm{CD}_{3} \mathrm{SSCD}_{3}\right)$ was observed at $m / z 100.0$ as expected (Fig. 4b). For the main fragment at $m / z 79\left(\mathrm{CH}_{3} \mathrm{SS}^{+}\right)$, its corresponding deuterated fragment was observed at $\mathrm{m} / z$ $82\left(\mathrm{CD}_{3} \mathrm{SS}^{+}\right)$(Fig. 4a versus Fig. 4b). For the minor fragments, the corresponding deuterium shifts were also generally observed; e.g., $m / z 61\left(\mathrm{CH}_{3} \mathrm{SCH}_{2}{ }^{+}\right)$versus $m / z 66\left(\mathrm{CD}_{3} \mathrm{SCD}_{2}{ }^{+}\right)$and $m / z, 45$ 
$\left(\mathrm{HCS}^{+}\right)$versus $m / z, 46\left(\mathrm{DCS}^{+}\right)$. Therefore, the observed deuterium shifts are consistent with the structure assignment of peak-4 as dimethyldisulfide.

For peak-3, it was proposed to be $S$-methyl methanethioate $\left[\mathrm{CH}_{3} \mathrm{SC}(\mathrm{O}) \mathrm{H}\right]$ as mentioned above. Its molecular ion of $\mathrm{m} / \mathrm{z} 76$ was also the base peak (Fig. 5a) and its deuterated counterpart was observed at $m / z 80$ as expected (Fig. 5b). Furthermore, for the major fragments at $\mathrm{m} / \mathrm{z} 47\left(\mathrm{CH}_{3} \mathrm{~S}^{+}\right)$and $\mathrm{m} / z 48\left(\mathrm{CH}_{3} \mathrm{SH}^{+}\right)$, their corresponding deuterated fragments were observed at $\mathrm{m} / z \quad 50\left(\mathrm{CD}_{3} \mathrm{~S}^{+}\right)$and $52\left(\mathrm{CD}_{3} \mathrm{SD}^{+}\right)$, respectively (Fig. 5a versus Fig. 5b).

\subsection{HS-GC-TOF MS analysis of peak-3}

The results obtained from the above HS-GC-MS study including the experiment with DMSO- $d_{6}$ suggested that peak-3 is $S$-methyl methanethioate. In order to confirm the proposed structure, high resolution HS-GC-MS analysis was conducted on a GC-QTOF MS instrument with headspace sampling (refer to Section 2.4). As shown in Fig. 5c, the fragmentation pattern of peak-3 was similar to that obtained on the GC-MS with single quadrupole MS detector (Fig. 5a). The accurate $m / z$ value of the molecule ion of peak-3 in the HS-GC-TOF MS analysis was 75.9976, matching the formula of $S$-methyl methanethioate $\left(\mathrm{C}_{2} \mathrm{H}_{4} \mathrm{OS}\right)$ with an error of $2.1 \mathrm{ppm}$. Moreover, the three fragments at $\mathrm{m} / \mathrm{z}$ value at $46.9950,48.0028$ and 44.9793 , match the formulas of $\mathrm{CH}_{3} \mathrm{~S}^{+}, \mathrm{CH}_{4} \mathrm{~S}^{+}$, and $\mathrm{CHS}^{+}$, respectively, all within an error of $5.0 \mathrm{ppm}$. The formation of these three fragments can be rationalized in Scheme 2.

\subsection{Stress study of methanthiol and formic acid in DMF}


It had been inferred above that peak-3 observed in the HS-GC-MS analysis is $S$-methyl methanethioate, which is one of the degradants derived from the minor decomposition of the sample diluent DMSO. In order to further confirm its structure, a stress study of mixing methanthiol and formic acid in DMF was performed. The solution prepared was loaded onto the HS-GC-MS instrument and analyzed under the same condition as the samples for residual solvent analysis (refer to Section 2.6). Peak-3 was generated as expected and its mass spectrum (Fig. 5d) is very similar to the original mass spectrum of peak-3 (Fig. 5a). Hence, peak-3 is further confirmed to be $S$-methyl methanethioate.

\subsection{Proposed degradation pathways of DMSO in HS-GC analyses}

All the evidence shown above demonstrates that the four impurities, i.e., methanethiol (peak-1), dimethylsulfide (peak-2), S-methyl methanethioate (peak-3), and dimethyldisulfide (peak-4) detected in the HS-GC analyses of several drug substances, are due to minor decomposition of the sample diluent DMSO. The structures of methanethiol (peak-1), dimethylsulfide (peak-2), and dimethyldisulfide (peak-4) are identified based on the high matching scores against the NIST MS library. For $S$-methyl methanethioate (peak-3), because its mass spectrum is not available in the NIST library, its structure is elucidated by a combination of the following approaches: plausible DMSO degradation chemistry, fragmentation pathways obtained from both the unit as well as high resolution GC-MS, and stress study of methanethiol and formic acid. According to our best knowledge, the mass spectrum of $S$-methyl methanethioate has not been reported in the literature. 
Based on the structures identified, the degradation pathways of DMSO can be proposed as follows (Scheme 1): First, under the heating condition of HS sampling $\left(80-140^{\circ} \mathrm{C}\right)$, DMSO may undergo an enolization-like process followed by 1,2-hydroxyl migration to form the key hydroxyldimethylsulfoxide intermediate. Second, the latter intermediate can dissociate to produce formaldehyde and methanethiol (peak-1). The methanethiol formed could be oxidized by DMSO to yield dimethyldisulfide (peak-4), while DMSO itself is reduced to become dimethylsulfide (peak-2). On the other hand, the hydroxyldimethylsulfoxide intermediate could also be oxidized to give $S$-methyl methanethioate (peak-3) by losing two hydrogens.

Several papers reported that DMSO can undergo degradation under very harsh conditions such as extreme heating (783 - 1,404K), microwaving, or advanced oxidation processing with hydroxyl radicals. ${ }^{16-21}$ Although these harsh conditions are not pharmaceutically relevant, these phenomena did give us a clue that DMSO might also undergo minor decomposition to produce these artifact peaks under the pharmaceutically relevant HS-GC conditions. On the other hand, in a study published by Tian and Rustum in 2016, an unknown peak was identified as dimethylsulfide in HS-GC residual solvent analysis of permethrin API when using DMSO as diluent, which was proposed to be formed via a redox reaction between DMSO and one of the impurities of permethrin, 3-phenoxylbenzyl chloride. ${ }^{22}$ Furthermore, in a recent study of our group, formaldehyde was present as an artifact peak in HS-GC analysis of several drug substances, resulting from decomposition of sample diluent DMSO. ${ }^{15}$ The results of these studies are consistent with the finding of the current study, i.e., 
DMSO can undergo minor decomposition in HS-GC analysis of certain drug substances to yield a few artifact peaks.

\section{Conclusions}

Through a comprehensive and systematic investigation, we have determined that the four unknown peaks in residual solvent testing of certain drug substances are due to minor degradation of DMSO used as sample diluent in the HS-GC analyses. In other words, the four peaks detected in these analyses are not real residual solvent impurities, but rather artifact peaks or ghost peaks. The structures of the four artifact peaks have also been determined and their plausible formation mechanisms proposed. The fact that these artifact peaks are observed in several structurally diverse drug substances suggests that formation of artifact peaks from DMSO could widely occur in HS-GC analysis, when DMSO is used as sample diluent. With the ever tightening regulatory requirements, small unknown peaks observed in GC analysis may need to be investigated. The finding in this paper should help avoid these artifact peaks or facilitate the root cause investigation, should such event occur in other pharmaceutical laboratories.

\section{ACKNOWLEDGMENTS}

We gratefully acknowledge the technical support by Ms. Lingyun Xu. 


\section{References}

1. International Conference on the Harmonisation of the Technical Requirements for Registration of Pharmaceuticals for Human Use (ICH) guideline Q3C (R6) on impurities: guideline for residual solvents, July 23, 2015, available on line: https://www.ema.europa.eu/en/ich-q3c-r6-residual-solvents\#current-effective-ver sion-section.

2. Nam J, Huang Y, Agarwal S, Lannutti J, Materials reflection and residual solvent retention in biodegradable electrospun fibers, $J$ Appl Polymer Sci. 2008;107(3):1547-1554.

3. Darvishmanesh S, Firoozpour L, Vanneste J, Luis P, Degrèvea J, Bruggena BV, Performance of solvent resistant nanofiltration membranes for purification of residual solvent in the pharmaceutical industry: experiments and simulation, Green Chem. 2011;13(12):3476-3483.

4. Grodowska K, Parczewski A, Organic solvents in the pharmaceutical industry, Acta Pol Pharm. 2010;67(1):3-12.

5. Liu H, Tang QL, Markovich RJ, Rustum AM, A general static-headspace gas chromatographic method for determination of residual benzene in oral liquid pharmaceutical products, J Pharm Biomed Anal. 2011;54(2):417-421.

6. Camarasu CC, Headspace SPME method development for the analysis of volatile polar residual solvents by GC-MS, J Pharm Biomed Anal. 2000;23(1):197-210.

7. Hymer CB, Residual solvent testing: a review of gas-chromatographic and alternative techniques, Pharm Res-Dordr. 2003;20(3)337-344. 
8. Grodowska K, Parczewski A, Analytical methods for residual solvents determination in pharmaceutical products, Acta Pol Pharm. 2010;67(1)13-26.

9. Penton Z, Determination of residual solvent in pharmaceutical preparations by static headspace GC, J High Resolut Chrom. 1992;15(5):329-331.

10. David F, Szücs R, Makwana J, Sandra P, Fast capillary GC using a low thermal mass column oven for the determination of residual solvents in pharmaceuticals, J Sep Sci. 2006;29(5):695-698.

11. Camarasu CC, Headspace SPME method development for the analysis of volatile polar residual solvents by GC-MS, J Pharm Biomed Anal. 2000;23(1):197-210.

12. Deconinck E, Canfyn M, Sacré PY, Baudewyns S, Courselle P, DeBeer JO, A validated GC-MS method for the determination and quantification of residual solvents in counterfeit tablets and capsules, J Pharm Biomed Anal. 2012;70: 64-70.

13. Bao QB, Fu KJ, Ren QQ, Zhong YY, Qian D, Accuracy profiles for analyzing residual solvents in textiles by GC-MS, J Sep Sci. 2017;55(9):882-890.

14. Lu Y, Zhu Y, Determination of chlorobenzenes in textiles by pressurized hot water extraction followed by vortex-assisted liquid-liquid microextraction and gas chromatography-mass spectrometry, J Chromatogr. A. 2013;1319(6):27-34.

15. Lin JS, Zhou Q, Zhu WQ, Li QM, Xu LY, Chen WB, Wang J, Zhang XH, Hu WP, Li M, Formation of formaldehyde as an artifact peak in head space GC analysis resulting from decomposition of sample diluent DMSO: A GC-MS investigation with deuterated DMSO, J Pharm Biomed Anal. 2020;188(5): 
113316.

16. Klein SM, Cohen G, Cederbaum AI, Production of formaldehyde during metabolism of dimethyl sulfoxide by hydroxyl radical generating systems, Biochemistry. 1981;20(21):6006-6012.

17. Carlsen L, Egsgaard H, Harpp D, Gas phase thermolyses. Part 4. Gas phase thermolyses of thietan 1-oxide and 1,2-oxathiolan 2-oxide. Evidence for the intermediacy of 1,2-oxathiolan, J.C.S. Perkin. II. 1981;12(8):1166-1170.

18. Lee $\mathrm{YH}$, Lee $\mathrm{CH}$, Yoon JY, Kinetics and mechanisms of DMSO (dimethylsulfoxide) degradation by $\mathrm{UV} / \mathrm{H}_{2} \mathrm{O}_{2}$ process, Water Res. 2004;38(10):2599-2588.

19. Mésangeau C, Yous S, Pérès B, Lesieur D, Besson T, Pictet-Spengler heterocyclizations via microwave-assisted degradation of DMSO, Tetrahedron Lett. 2005;46(14):2465-2468.

20. Wang JM, Rochon FD, Yang Y, Hua L, Kayser MM, Synthesis of oxazolidines using $\mathrm{DMSO} / \mathrm{P}_{4} \mathrm{O}_{10}$ as a formaldehyde equivalent, Tetrahedron-Asymmetr. 2007;18(9):1115-1123.

21. Li Z, Xu XY, Peng YQ, Jiang ZX, Ding CY, Qian XH, An unusual synthesis of Tr öger's bases using DMSO/HCl as formaldehyde equivalent, ChemInform. 2005; 36(8):1228-1230.

22. Tian JZ, Rustum AM, Investigation of dimethyl sulfide formation during GC analysis of permethrin API: study of the reaction kinetics and estimation of the activation energy of the reaction, $R S C A d v .2016 ; 86(6): 83020-83024$. 
(a)

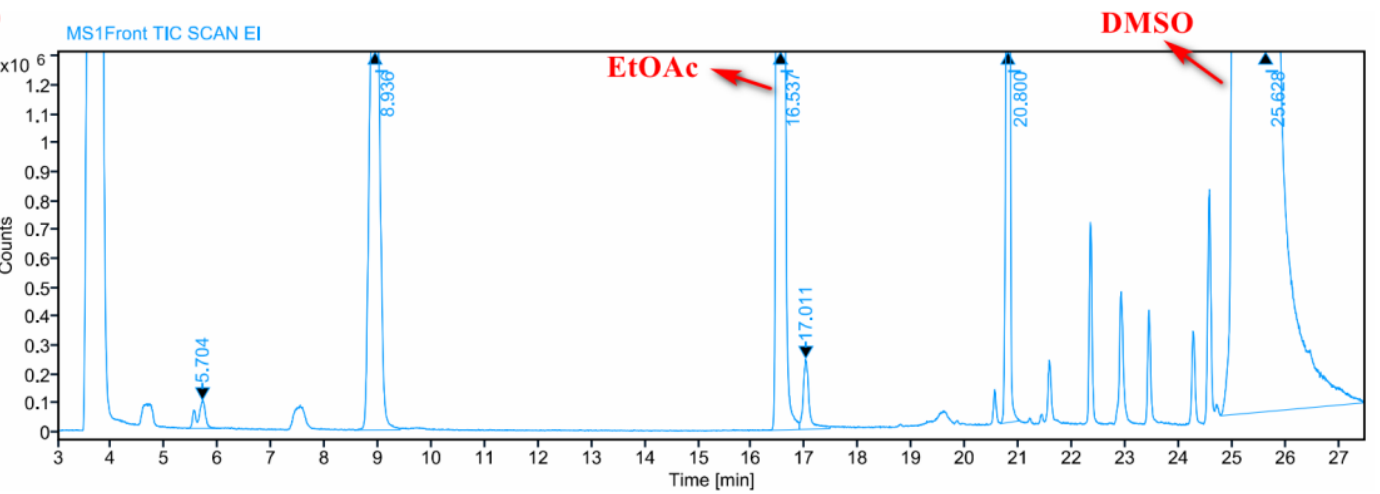

(b)

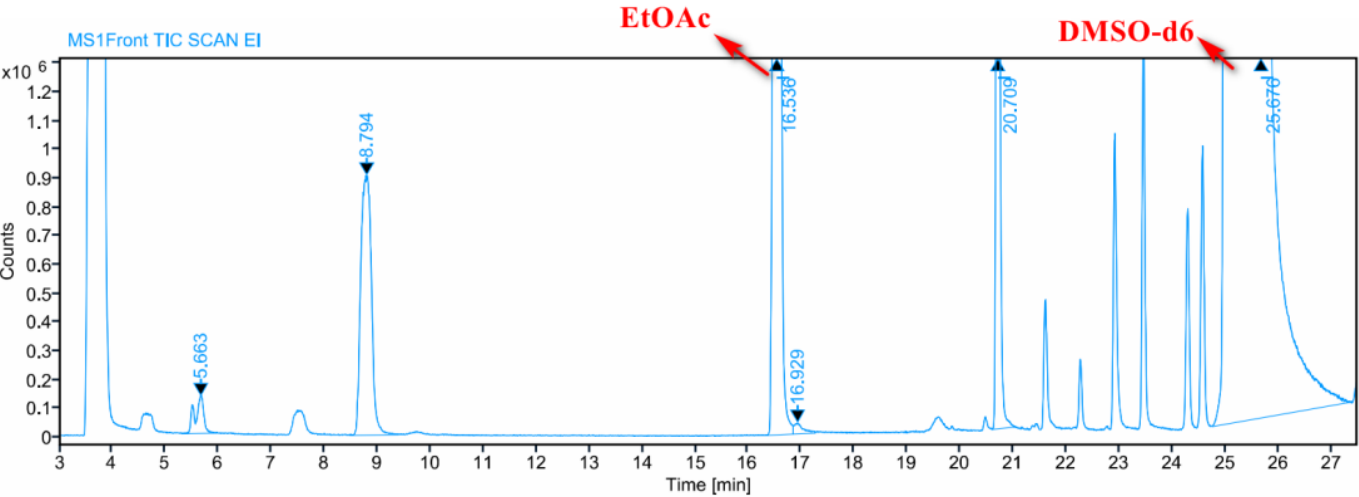

(c)

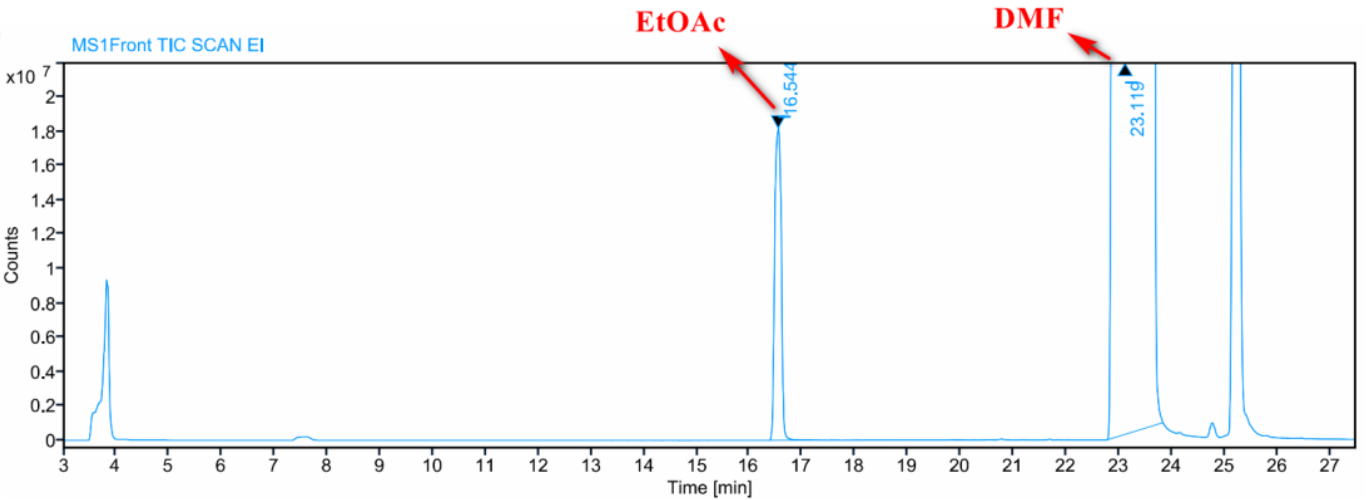

Fig. 1. (a) TIC chromatogram of a drug substance (using DMSO as diluent) in HS-GC-MS analysis. The four impurities which are derived from DMSO eluted at 5.704, 8.936, 17.011, and $20.800 \mathrm{~min}$, respectively. Ethyl acetate is the real residual solvent in the drug sbustance. (b) TIC chromatogram of the drug substance (using DMSO- $d_{6}$ as diluent) in HS-GC-MS analysis. The four impurities which are derived from DMSO- $d_{6}$ eluted at 5.663, 8.794, 16.929, and $20.709 \mathrm{~min}$, respectively. The elution profiles in their TIC chromatograms are very similar. (c) TIC chromatogram of the drug substance in HS-GC-MS analysis using DMF as diluent. 

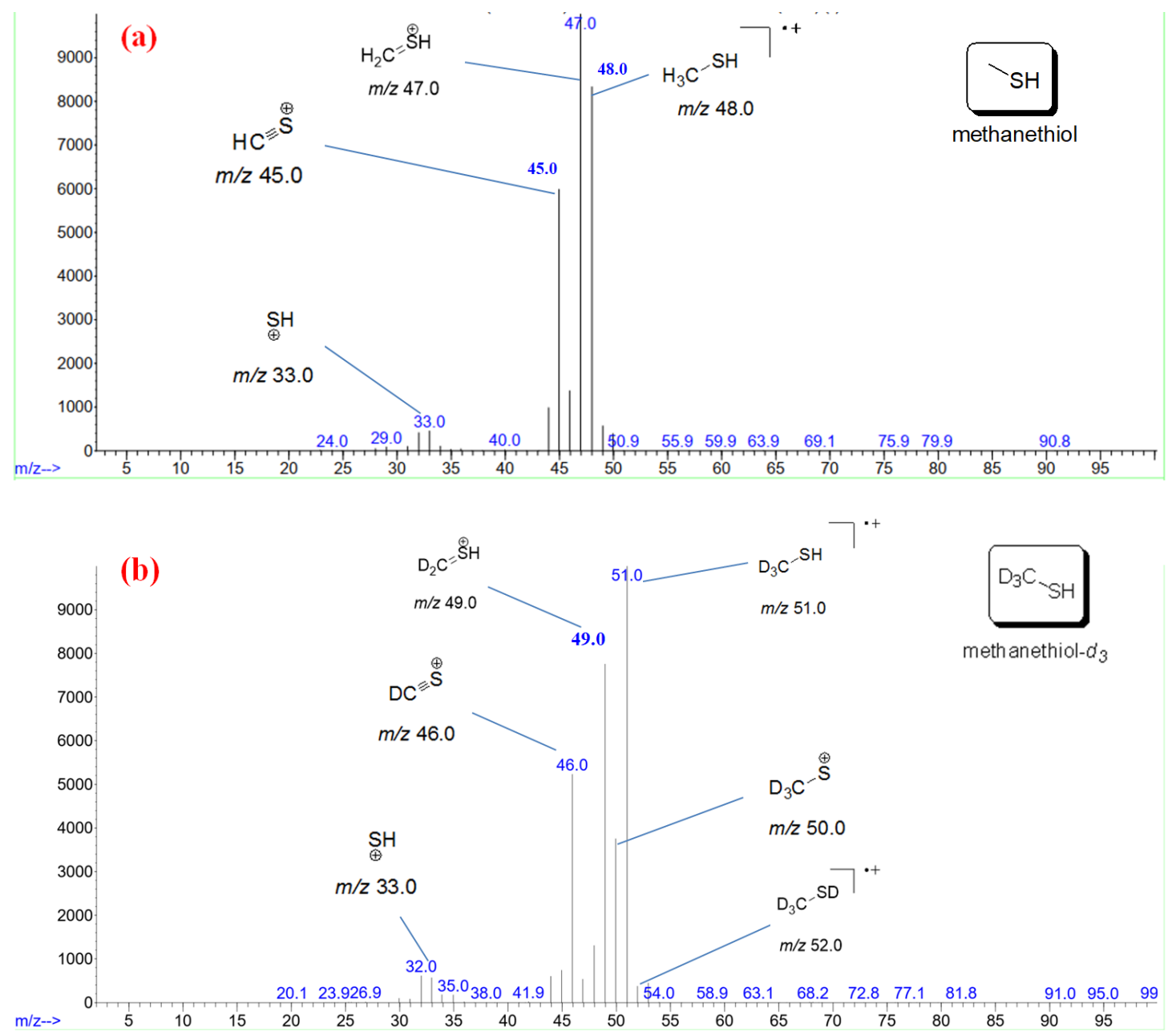

Fig. 2. (a) MS spectrum of 5.704 min species (peak-1) in residual solvent analysis in a drug substance (using DMSO as diluent), which is confirmed to be methanethiol. (b) MS spectrum of peak-1 in residual solvent analysis in a drug substance (using DMSO- $d_{6}$ as diluent), which is confirmed to be methanethiol- $d_{3}$. 

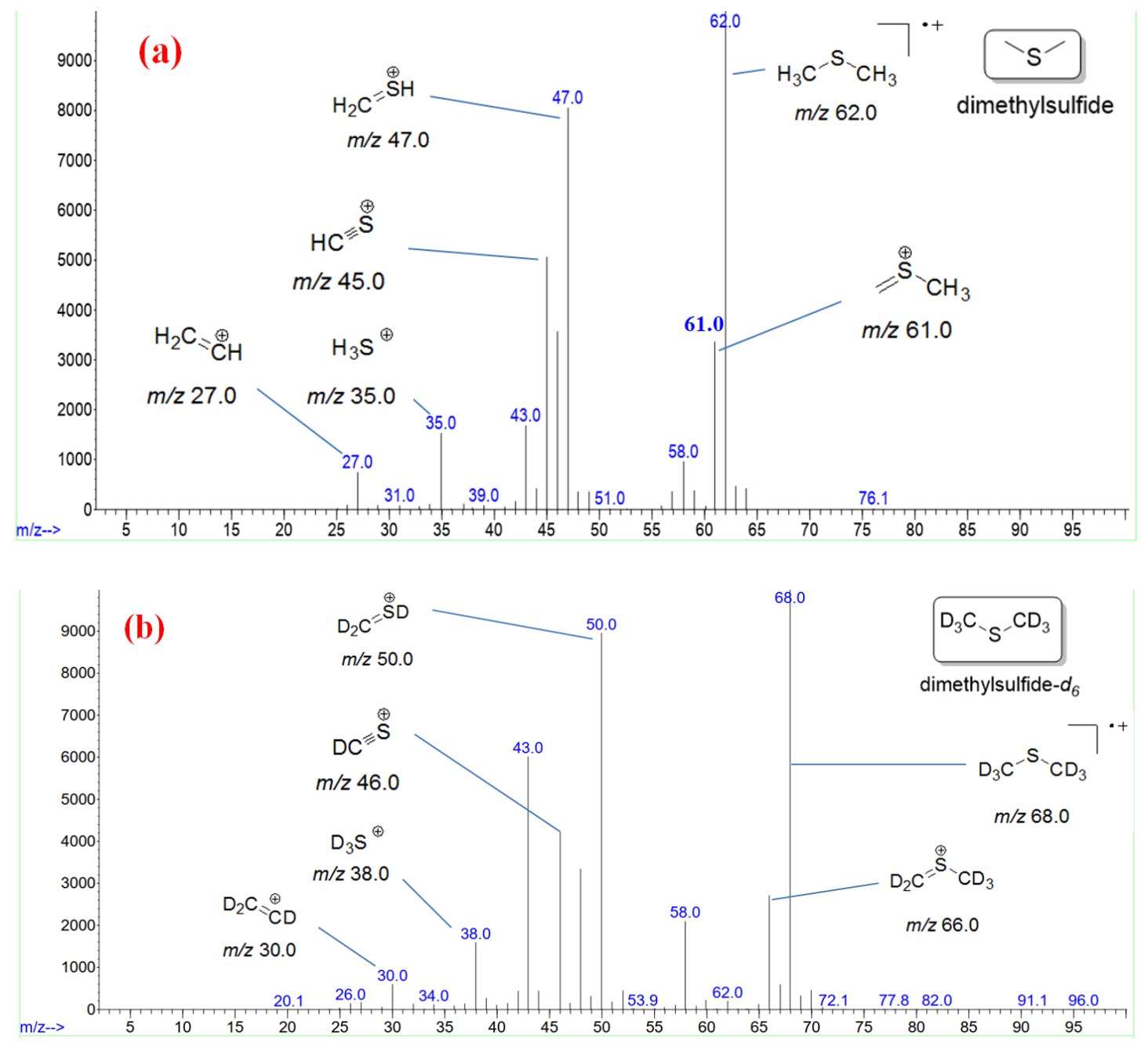

Fig. 3. (a) MS spectrum of 8.936 min species (peak-2) in residual solvent analysis in a drug substance (using DMSO as diluent), which is confirmed to be dimethylsulfide. (b) MS spectrum of peak-2 in residual solvent analysis in a drug substance (using DMSO- $d_{6}$ as diluent), which is confirmed to be dimethylsulfide- $d_{6}$. The fragaments shown at $\mathrm{m} / \mathrm{z} 43, \mathrm{~m} / \mathrm{z}, 48$ and $\mathrm{m} / \mathrm{z}, 58$ are found to originate from background interference. 

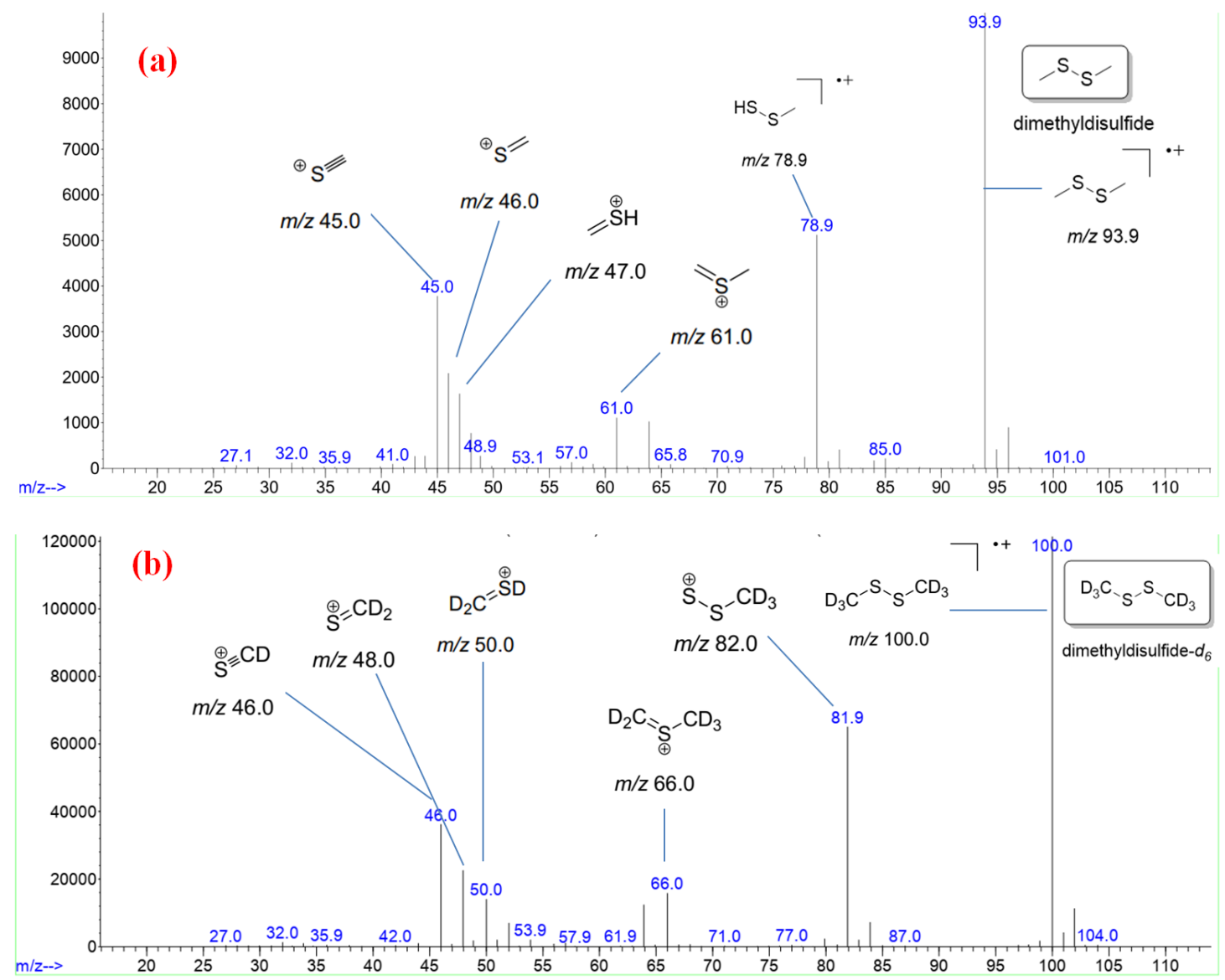

Fig. 4. (a) MS spectrum of 20.800 min species (peak-4) in residual solvent analysis in a drug substance (using DMSO as diluent), which is confirmed to be dimethyldisulfide. (b) MS spectrum of peak-4 in residual solvent analysis in a drug substance (using DMSO- $d_{6}$ as diluent), which is confirmed to be dimethyldisulfide- $d_{6}$. 

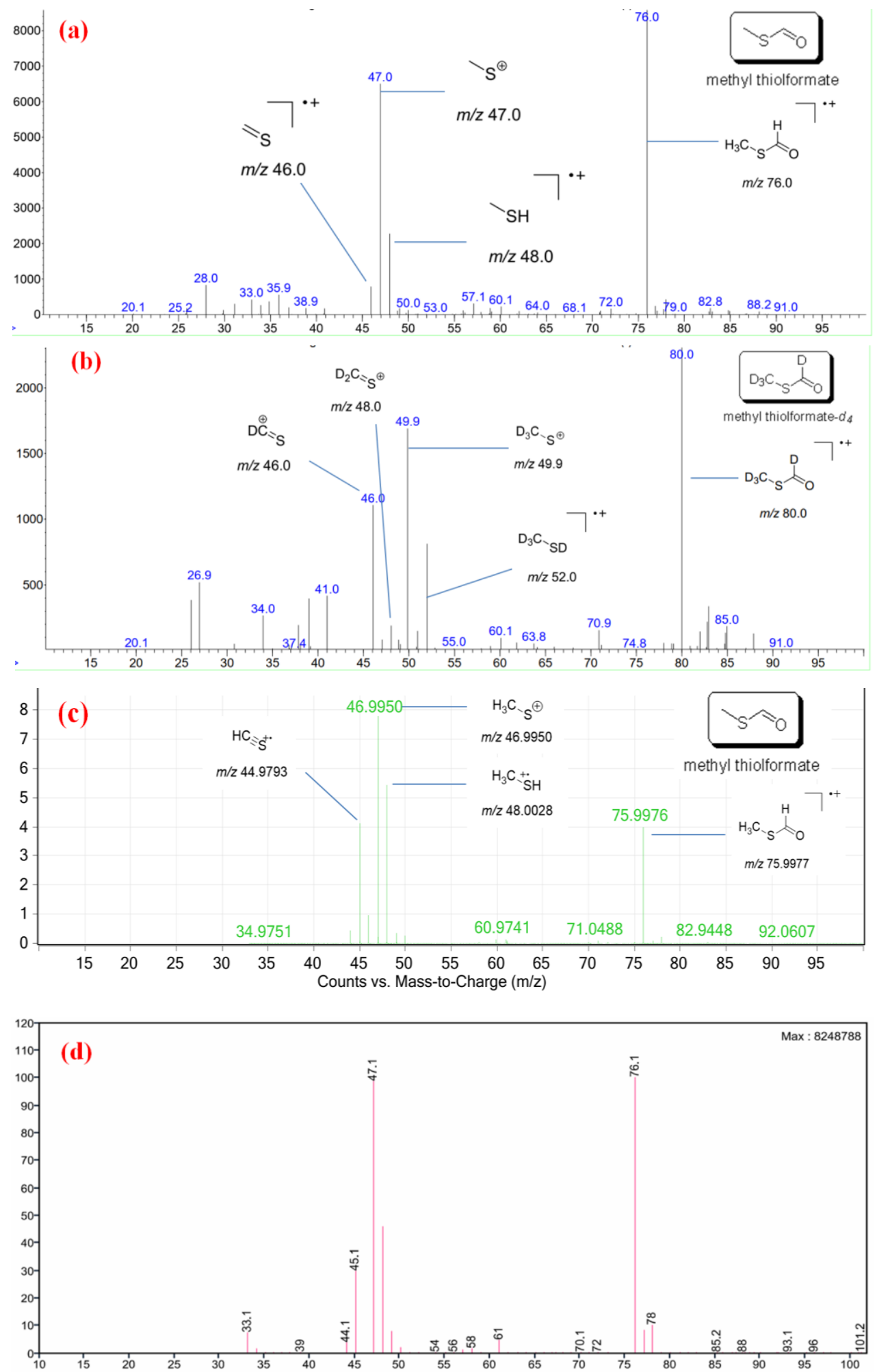

Fig. 5. EI-MS spectra of impurities observed in HS-GC-MS analyses for residual solvent detection in certain drug substances with DMSO as sample diluent: (a) MS spectrum of 17.011 min species (peak-3) analyzed using regular DMSO as diluent. (b) MS spectrum of peak-3 analyzed using DMSO- $d_{6}$ as diluent. The fragaments shown at $m / z$ 82.9, 84.9, 61.9, 39.9, and 29.9 are found to be background interference. (c) High resolution MS spectrum of $17.011 \mathrm{~min}$ species (peak-3) analyzed using DMSO as diluent. (d) MS spectrum of the degradant obtained from the stress study of methanthiol and formic acid in DMF. 


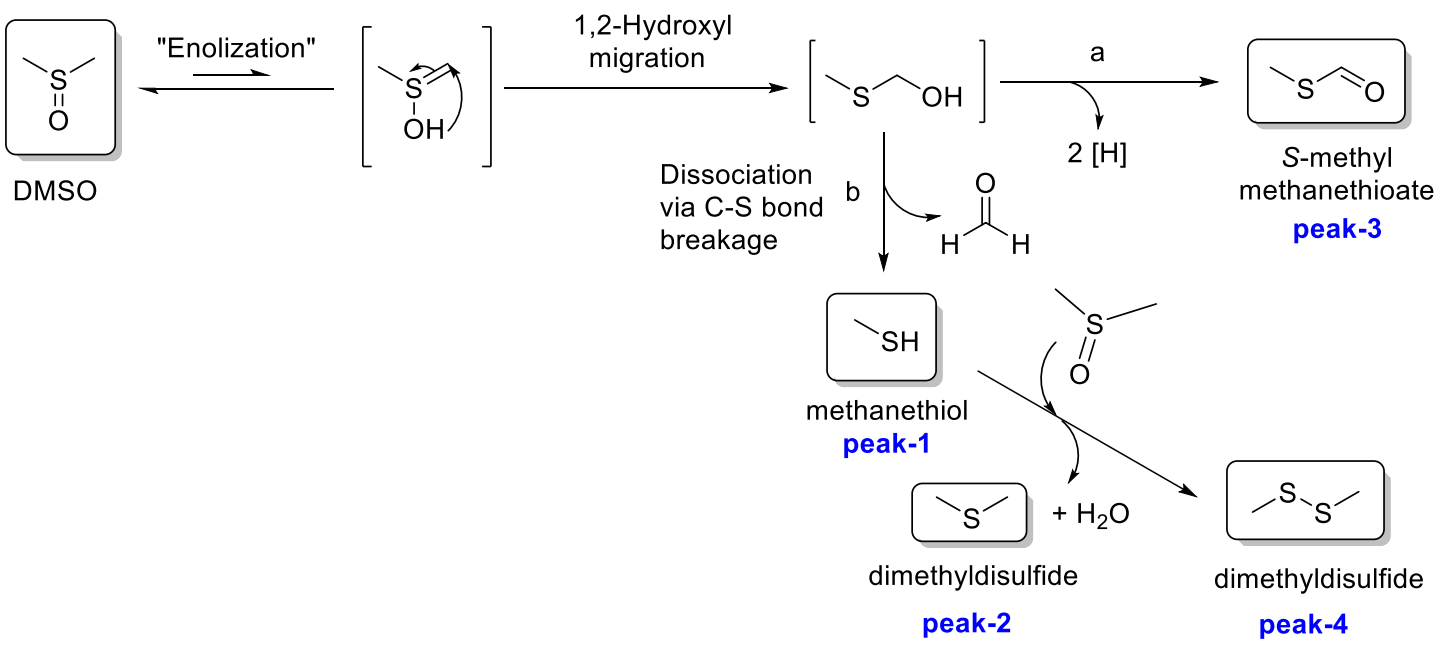

Scheme 1. Plausible degradation pathways of DMSO in HS-GC analyses based on the current study.

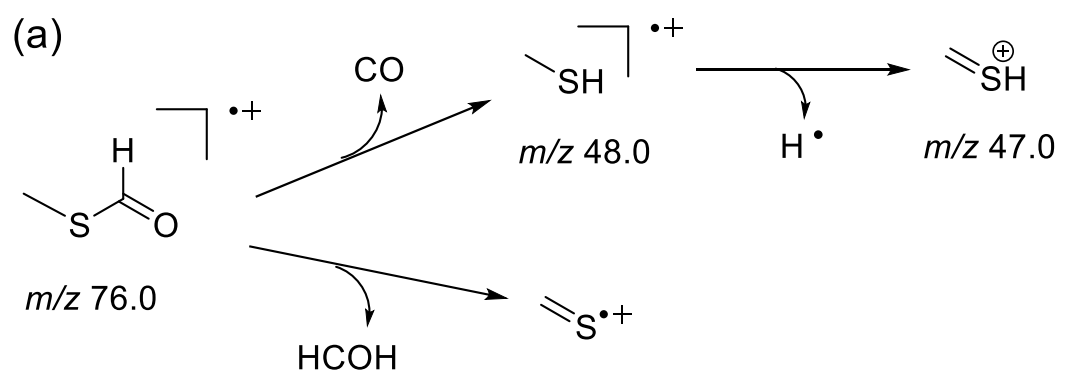

$m / z 46.0$

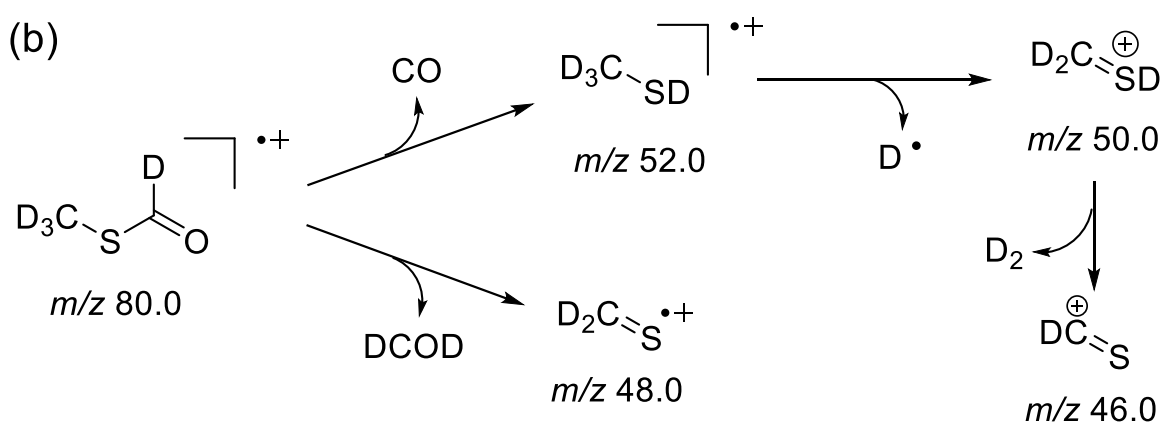

Scheme 2. Proposed mechanisms for the fragmentation pathways of $S$-methyl methanethioate (a) and $S$-methyl methanethioate- $d_{4}$ (b) in EI-MS. 
Table 1. Stuctures and deuterium mass shift $(\mathrm{m} / \mathrm{z})$ of the four degradants of DMSO in HS-GC-MS residual solvent analysis of certain drug substances.

\begin{tabular}{|c|c|c|c|c|c|}
\hline No. & $\begin{array}{l}\text { Rention } \\
\text { time (min) }\end{array}$ & Name & Stucture & $\begin{array}{l}\text { Isotope } m / z \\
\text { shift of the } \\
\text { molecule ion }{ }^{1}\end{array}$ & $\begin{array}{c}\text { Matching score } \\
\text { with NIST } \\
\text { library }^{2}\end{array}$ \\
\hline 1 & 5.704 & Methanethiol (peak-1) & & $+3^{3}$ & 91 \\
\hline 2 & 8.936 & $\begin{array}{c}\text { Dimethylsulfide } \\
\text { (peak-2) }\end{array}$ & & +6 & 95 \\
\hline 3 & 17.011 & $\begin{array}{c}S \text {-methyl } \\
\text { methanethioate } \\
\text { (peak-3) }\end{array}$ & $\mathrm{O}$ & +4 & Not available \\
\hline 4 & 20.800 & $\begin{array}{c}\text { Dimethyldisulfide } \\
\text { (peak-4) }\end{array}$ & & +6 & 97 \\
\hline
\end{tabular}

\section{Notes:}

1: The HS-GC-MS experiments with deuterium isotope mass shift were conducted using DMSO- $d_{6}$ as the diluent.

2: The matching scores are the matching results between the four degradants generated from regular DMSO and the mass spectra available in the NIST MS library, respectively.

3: A D/H exchange apparently occurred with the $\mathrm{CD}_{3}-\mathrm{SD}$ (methanethiol- $d_{4}$ ) generated from DMSO- $d_{6}$; the sources of $\mathrm{H}$ are probably resiudal water in DMSO and that generated during the course of DMSO decomposition (Scheme 1). 